\title{
NUTRITIONAL STATE OF HCV-INFECTED INDIVIDUALS EXAMINED AND TREATED IN OUTPATIENT CLINIC
}

THESIS. C. M. Carreira submitted this dissertation for her Masters in Tropical Diseases at Botucatu School of Medicine, São Paulo State University - UNESP, Botucatu, São Paulo, Brazil, 2004.

Advisor: Professor Paulo Câmara Marques Pereira

ABSTRACT: Hepatitis C affects more than 170 million people in the world. It is considered a severe health problem with 70 to $80 \%$ of the cases showing a chronic evolution. The hepatic lesion caused by the $\mathrm{C}$ virus (HCV) can interfere in the patients' nutritional state and contribute to the progress of the hepatic disease to cirrhosis (20\% of the cases). From the eighty-two adult individuals clinically examined, $76.8 \%$ were men, with mean age of 37.2 years. They were subdivided into 4 groups: Control Group (CG), with 26 healthy individuals; Group 1 (G1), with $30 \mathrm{HCV}$-infected individuals that did not need drug treatment; Group 2 (G2), with 14 HCV-infected individuals in treatment; and Group 3 (G3), with 12 individuals infected by HCV 12 months after the end of the treatment. All groups were investigated according to clinical-epidemic factors (transmission mechanism, social and economical condition, sedentary life, smoking, and alcohol abuse); laboratory characteristics; family history; eating habits; drug treatment; and anthropometric measures, such as body weight, body mass index (BMI), and evaluation of corporeal fat and thin mass. These last two parameters were eutrophic for values between 10 and $90 \%$. The epidemiological factors (alcoholism and sedentary life) did not present statistical difference between groups, only smoking showed a significant difference in G1. The actual weight of all individuals was higher than the expected according to sex, age and hepatic disease. The BMI showed slight obesity according to the World Health Organization (WHO), 1998. The HCV-infected individuals presented an 
increased percentage of body fatness $(24.6 \% \pm 8.7,27.2 \% \pm 9.7$, and $29.1 \% \pm 9.8$, respectively). Therefore, the anthropometric indicators of adipose mass showed that the reserves were preserved, possibly being associated to smoking and sedentary habits, which lead to coronary diseases, hyperlipidemia and arteriosclerosis. According to the thin mass evaluation, most of the HCV-infected individuals were eutrophic, not showing nutritional risk. The analysis of the diet's macronutrients showed that the energetic and proteic consumes were made according to the specific recommendation to individuals with hepatitis C. On the other hand, the quantity of lipids in the diet was 10 to $15 \%$ higher than the recommended to the individuals of Group 1. The percentage of saturated fat was high in the three groups studied. In view of the obtained results, a nutritional evaluation shows to be necessary in HCV-infected individuals, for posterior intervention and prevention of nutritional problems.

KEY WORDS: Hepatitis C, nutritional state.

\section{CORRESPONDENCE TO:}

C. M. CARREIRA, Departamento de Doenças Tropicais e Diagnóstico por Imagem, Faculdade de Medicina de Botucatu, UNESP, Distrito de Rubião Junior, s/n, 18618-000, Botucatu, SP, Brasil. Phone: 55143811 6212. Email: ppereira@fmb.unesp.br 